\section{Acondicionamento e coleta de resíduos sólidos domiciliares e impactos na saúde de crianças residentes em assentamentos periurbanos de Salvador, Bahia, Brasil}

\author{
Household solid waste bagging and collection \\ and their health implications for children living in \\ outlying urban settlements in Salvador, Bahia \\ State, Brazil
}

Luiz Roberto Santos Moraes 1

\section{Introdução}

This paper presents a study on the bagging and collection of household solid waste and the health implications for children. The research was conducted in nine human settlements on the outskirts of Salvador, Bahia State, Brazil. Intestinal nematode infection, predominantly involving Ascaris lumbricoides, Trichuris trichiura, and hookworms, was used as an epidemiological indicator in 1,893 children from 5 to 14 years of age. The study also included diarrhea incidence and nutritional status as shown by anthropometric indicators in 1,204 children less than 5 years of age. There was a higher prevalence of the three nematodes in children living in households without proper bagging/isolation and collection of household solid waste as compared to those in areas with regular garbage collection and adequate isolation of solid waste. The differences were statistically significant when other socioeconomic, cultural, demographic, and environmental risks factors were considered in the analysis. Similar results were also observed for epidemiological indicators, diarrhea incidence, and nutritional status.

Diarrhea; Nematode Infections; Solid Waste Conditioning; Solid Waste Collection
Alguns autores consideram bastante clara a relação entre a saúde pública e o acondicionamento, a coleta e a disposição de resíduos sólidos 1 . Outros autores 2,3,4 consideram os resíduos sólidos como um dos determinantes da estrutura epidemiológica da comunidade, exercendo sua ação sobre a incidência das doenças, ao lado de outros fatores.

Do ponto de vista sanitário, a importância dos resíduos sólidos como causa direta de doenças não está muito comprovada. Porém, como fator indireto os resíduos sólidos têm grande importância na transmissão de doenças como, por exemplo, por meio de vetores como artrópodes - moscas, mosquitos, baratas - e roedores que encontram nos resíduos sólidos alimento e condições adequadas para proliferação 2,3,4,5,6.

Segundo a Fundação Nacional de Saúde 7, os resíduos sólidos constituem importante problema sanitário quando não recebem os devidos cuidados e as medidas adotadas para sua solução adequada têm, sob o aspecto sanitário, objetivo comum a outras medidas de saneamento ambiental, como prevenir e controlar doenças a eles relacionadas.

Estudo da Organização Pan-Americana da Saúde conclui que a correta solução do problema dos resíduos sólidos resulta na redução de $90 \%$ das moscas, $65 \%$ dos ratos e $45 \%$ dos mosquitos 8 
Zanon 9 observa que o risco potencial de transmissão direta de doenças infecciosas por qualquer tipo de resíduo sólido dependerá: (i) da presença de um agente infeccioso; (ii) da sua capacidade de sobrevivência no resíduo sólido; e (iii) da possibilidade de sua transmissão para um hospedeiro susceptível.

Contudo, estudos de associação entre manejo, coleta e disposição inadequados de resíduos sólidos e morbidade infantil não são de fácil realização, além de serem escassos na literatura 10,11,12,13, e os resultados dependem de um número considerável de outros fatores para sua interpretação adequada.

O presente artigo tem como objetivo estudar o acondicionamento e coleta dos resíduos sólidos domiciliares e os impactos na saúde de crianças, por meio de estudo desenvolvido em assentamentos periurbanos da cidade de Salvador, Bahia, Brasil, entre julho de 1989 e dezembro de 1990.

\section{Materiais e métodos}

A escolha dos indicadores epidemiológicos para avaliar o impacto na saúde de crianças foi feita após revisão da literatura sobre o assunto, sendo utilizados a morbidade por doença diarréica expressa pela incidência de diarréia (número de episódios por criança/ano), o estado nutricional expresso por meio de indicadores antropométricos (escores-z de altura/idade, de peso/idade e de peso/altura) e a infecção por nematóides intestinais expressa pela prevalência de Ascaris lumbricoides, Trichuris trichiura e ancilostomídeos.

O impacto na saúde de crianças devido aos resíduos sólidos foi estudado de duas formas: uma considerando o domínio doméstico, expresso pelo tipo de acondicionamento domiciliar dos resíduos sólidos, e outra considerando o domínio público, expresso pela coleta de resíduos sólidos domiciliares. Para a primeira, considerou-se como variável independente principal o tipo de acondicionamento domiciliar dos resíduos sólidos, definida em três categorias: adequado (saco plástico, lata ou balde com tampa), inadequado (caixa de papelão, caixote de madeira ou balde sem tampa) e sem acondicionamento; e para a segunda, considerou-se como variável independente principal a coleta dos resíduos sólidos domiciliares no ambiente público, definida em três categorias: regular (diária, alternada ou semanal podendo ser porta-a-porta, de caixa estacionária ou de ponto de lixo), irregular (mensal ou esporádica de caixa estacionária ou ponto de lixo) e não coletado (vazado em terreno baldio, canal ou quintal).
O Projeto AISAM (Avaliação do Impacto de Medidas de Saneamento Ambiental em Áreas Pauperizadas de Salvador), realizado pelo Departamento de Engenharia Ambiental da Escola Politécnica da Universidade Federal da Bahia, entre julho de 1989 e dezembro de 1990, em nove assentamentos humanos periurbanos da cidade, embora tendo como objetivo principal avaliar o efeito sobre a saúde de crianças de soluções de esgotamento sanitário e drenagem de águas pluviais, oportunizou também a coleta de dados de outros fatores do ambiente doméstico e do ambiente comunitário, incluindo o acondicionamento e a coleta dos resíduos sólidos domiciliares, além de fatores demográficos, culturais e sócio-econômicos 14. O projeto foi financiado pelo International Development Research Centre (IDRC) do Canadá.

Dessa forma, pode-se analisar os dados coletados sobre os resíduos sólidos domiciliares estudando-se sua associação com alguns indicadores epidemiológicos. A população de estudo foi formada por crianças menores de 15 anos de idade residentes nos nove assentamentos humanos selecionados. As crianças menores de cinco anos de idade foram acompanhadas durante um ano num estudo longitudinal para estimar a incidência de diarréia, e em estudos seccionais espaçados de dois em dois meses para medição de peso e de altura. As de idade entre 5 e 14 anos foram observadas em três estudos seccionais, sendo dois deles para avaliar a reinfecção por nematóides intestinais 14 .

Com o objetivo de coletar as histórias de diarréia das 1.204 crianças menores de cinco anos de idade participantes do estudo, adotouse um sistema de registro diário pelas mães por meio de "calendário" quinzenal desenhado especialmente para esta finalidade. As mães foram estimuladas a relembrar diariamente com sinal de positivo (+) ou de negativo (-) se cada uma das suas crianças tiveram ou não diarréia naquele dia.

Além disso, as mães foram entrevistadas sobre episódios de diarréia nas suas crianças. As entrevistas foram realizadas em intervalos quinzenais, no fim dos quais, o calendário preenchido foi coletado e um novo entregue à mãe.

Visando a estudar o estado nutricional das crianças e a sua possível associação com a variáveis tipo de acondicionamento domiciliar e coleta dos resíduos sólidos domiciliares, foram realizadas medições antropométricas nas mesmas crianças observadas quanto à diarréia. $\mathrm{O}$ peso foi medido mensalmente por meio de balança portátil com precisão de \pm 100 g e a estatura medida de dois em dois meses, utilizando-se antropômetro de madeira com precisão de $\pm 0,5 \mathrm{~cm}$. 
Amostras para exame parasitológico de fezes foram coletadas em 1.893 crianças entre 5 e 14 anos, com particular atenção para identificação e contagem de ovos dos três nematóides estudados, por meio da técnica de Kato como descrito por Katz et al. 15.

Informações sobre um grande número de variáveis intervenientes sócio-econômicas, culturais e demográficas, e de outras variáveis ambientais, além da coleta de lixo, foram também coletadas para fins de controle quando das análises estatísticas.

Os dados foram armazenados em microcomputador utilizando-se o programa dBASE III+ (Ashton-Tate Corp., Estados Unidos) e analisados com o pacote estatístico SPSS/PC+ versão 4.01 (SPSS Inc., Chicago, Estados Unidos). Os dados de peso e estatura foram transformados em indicadores antropométricos com a utilização do programa CASP Anthropometric Software Package version 3.0 (Centers for Disease Control and Prevention, Atlanta, Estados Unidos). Cálculos de prevalência de nematóides, incidência de diarréia, escores-z de altura/idade, peso/idade e peso/altura, testes de qui-quadrado e análises de variância e de regressão logística (esta última apenas para o indicador epidemiológico - prevalência dos nematóides intestinais e variável de exposição de domínio público - coleta de resíduos sólidos) foram realizados.

\section{Resultados e discussão}

A Tabela 1 mostra a associação estatisticamente significante $(p<0,00001)$ entre a variável acondicionamento domiciliar de resíduos sólidos e a prevalência dos nematóides estudados, notando-se maiores prevalências para as crianças residentes em domicílios sem acondicionamento quando comparadas com aquelas residentes em domicílios onde os resíduos sólidos são adequadamente acondicionados.

Como pode ser observado na Tabela 2, houve também uma associação estatisticamente significante $(p<0,001)$ entre acondicionamento domiciliar de resíduos sólidos e incidência de diarréia.

Em relação ao estado nutricional, as crianças residentes em domicílios sem acondicionamento dos resíduos sólidos apresentaram menores escores- $z$ de altura/idade $(p<0,001)$ e de peso/ idade $(\mathrm{p}<0,05)$ que aquelas de domicílios que dispunham de acondicionamento categorizado como adequado ou inadequado (Tabela 3 ).

A Tabela 4 apresenta a prevalência de A. lumbricoides, T. trichiura e ancilostomídeos sempre maior nas crianças de domicílios que não dispõem de coleta de resíduos sólidos e sempre menor nas crianças residentes em domicílios que dispõem de coleta regular. O teste de tendência para todos os três nematóides apresenta resultados estatísticos altamente significantes $(\mathrm{p}<0,00001)$.

Tabela 2

Tipo de acondicionamento domiciliar de resíduos sólidos e incidência de diarréia ( $\mathrm{n}=1.017)$.

\begin{tabular}{lcc}
\hline Tipo de acondicionamento & $\%$ & $\begin{array}{c}\text { Episódios por criança/ } \\
\text { ano *,** }\end{array}$ \\
\hline Adequado & 57,6 & 2,54 \\
Inadequado & 32,5 & $4,18 *, \star \star \star$ \\
Sem acondicionamento & 9,9 & $5,49 *$
\end{tabular}

* $p<0,001$;

** Análise de variância unidirecional usada para comparação de incidências médias: valor de p apresentado acima;

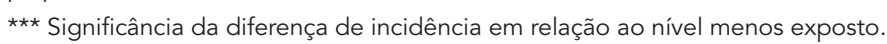

Tabela 1

Tipo de acondicionamento domiciliar de resíduos sólidos e infecções com nematóides intestinais $(n=1.893)$.

\begin{tabular}{|c|c|c|c|c|c|c|c|}
\hline \multirow[t]{2}{*}{ Tipo de acondicionamento } & \multirow[t]{2}{*}{$\mathbf{N}$} & \multicolumn{2}{|c|}{ Ascaris } & \multicolumn{2}{|c|}{ Trichuris } & \multicolumn{2}{|c|}{ Ancilóstomo } \\
\hline & & $\mathbf{n}$ & $\%$ * & $\mathbf{n}$ & $\%$ ** & $\mathbf{n}$ & $\%$ *** \\
\hline Adequado & 1.062 & 450 & 42,9 & 731 & 68,8 & 108 & 10,2 \\
\hline Inadequado & 691 & 407 & 58,9 & 583 & 84,4 & 130 & 18,8 \\
\hline Sem acondicionamento & 140 & 99 & 70,7 & 124 & 88,6 & 34 & 24,3 \\
\hline
\end{tabular}

* $\chi^{2} \mathrm{MH}=69,77 ; \mathrm{p}<0,00001$;

** $\chi^{2 \mathrm{MH}}=64,31 ; \mathrm{p}<0,00001$;

*** $\chi^{2} \mathrm{MH}=36,901 ; \mathrm{p}<0,00001$. 
Tipo de acondicionamento domiciliar de resíduos sólidos e estado nutricional ( $n=1.074)$.

\begin{tabular}{lccc}
\hline Tipo de acondicionamento & Altura/ldade & $\begin{array}{c}\text { Indicadores antropométricos } \\
\text { Peso/ldade }\end{array}$ & $\begin{array}{c}\text { Peso/Altura } \\
\text { Adequado }\end{array}$ \\
\hline Inadequado & $-0,62$ & $-0,45$ & $-0,10$ \\
Sem acondicionamento & $-0,80$ & $-0,53$ & $-0,08$ \\
Valor de p * & $-1,24$ & $-0,86$ & $-0,15$ \\
& $<0,001$ & $<0,05$ & $*$ \\
\hline
\end{tabular}

* Análise de variância unidirecional usada para comparação de médias;

** Não significante $(p>0,05)$.

Tabela 4

Coleta de resíduos sólidos domiciliares e infecções com nematóides intestinais ( $n=1.893$ ).

\begin{tabular}{|c|c|c|c|c|c|c|c|}
\hline \multirow[t]{2}{*}{ Coleta de resíduos sólidos } & \multirow[t]{2}{*}{$\mathbf{N}$} & \multicolumn{2}{|c|}{ Ascaris } & \multicolumn{2}{|c|}{ Trichuris } & \multicolumn{2}{|c|}{ Ancilóstomo } \\
\hline & & $\mathrm{n}$ & $\%$ * & $\mathrm{n}$ & $\%$ ** & $\mathrm{n}$ & $\% * \star \star$ \\
\hline Regular & 590 & 242 & 41,0 & 391 & 66,3 & 58 & 9,8 \\
\hline Irregular & 614 & 266 & 43,3 & 449 & 73,1 & 52 & 8,5 \\
\hline Não coletado & 689 & 448 & 65,0 & 598 & 86,8 & 162 & 23,5 \\
\hline
\end{tabular}

* $\chi^{2} \mathrm{MH}=76,36 ; \mathrm{p}<0,00001$

${ }^{\star *} \chi^{2} \mathrm{MH}=76,64 ; \mathrm{p}<0,00001$;

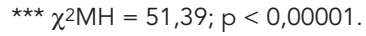

Os fatores de risco que mostraram associação estatisticamente significativa quando analisados isoladamente com a prevalência dos nematóides estudados como, idade e sexo da criança, número de moradores no domicílio, aglomeração (número de pessoas/cômodo), renda mensal familiar per capita, religião, presença de lavatório, piso do domicílio e disposição dos excretas humanos/esgotos sanitários, foram selecionados como variáveis potencialmente confundidoras para inclusão nas análises multivariadas de regressão logística.

Considerando o odds ratio igual a 1, para a coleta regular de resíduos sólidos domiciliares, os valores obtidos com os respectivos intervalos de confiança a 95\% para coleta irregular e não coletado foram 1,10 (1,09-1,11) e 2,67 (2,37-3,00) para A. lumbricoides, 1,38 (1,31-1,45) e 3,34 (2,90-3,85) para $T$. trichiura e $0,85(0,83-0,87)$ e $2,82(2,39$ $3,33)$ para ancilostomídeos, respectivamente.

Quando esses valores de odds ratio foram ajustados considerando as variáveis potencialmente confundidoras, eles tornaram-se 1,05 $(1,04-1,06)$ e $1,89(1,63-2,19)$ para A. lumbricoides e $1,20(1,13-1,28)$ e $1,91(1,62-2,25)$ para
T. trichiura, podendo então ser notado que após o ajuste, o odds ratio estimado é um pouco menor em relação ao não ajustado e que a associação entre a prevalência e coleta de resíduos sólidos continua estatisticamente altamente significante ( $\mathrm{p}<0,0001$ ) para estes dois nematóides. Para ancilostomídeos, os odds ratio ajustados passaram para $0,77(0,73-0,81)$ e $1,89(1,56-2,29)$.

As crianças residentes em domicílios/logradouros sem coleta de resíduos sólidos apresentam maior incidência de diarréia que as residentes em domicílios com coleta irregular, e aquelas residentes em domicílios com coleta regular apresentam menor incidência de diarréia, sendo que o teste de tendência apresenta resultado estatístico altamente significante $(\mathrm{p}<0,001)$ (Tabela 5). Achado similar foi encontrado por Heller 16, Catapreta \& Heller 17 e Rêgo et al. 18. A relação de densidade de incidência entre as crianças residentes em domicílios/logradouros com coleta regular e sem coleta foi de 0,35 e de 0,57 entre coleta irregular e sem coleta, significando que as crianças residentes em domicílios/logradouros com coleta regular e irregular experimentaram, respectivamente, $65 \%$ e $43 \%$ menor número de 
episódios de diarréia que aquelas que residiam em locais sem coleta de resíduos sólidos.

Quanto ao estado nutricional, as crianças residentes em domicílios/logradouros sem coleta dos resíduos sólidos apresentaram menores escores- $z$ de altura/idade $(p<0,001)$ e de peso/ idade $(\mathrm{p}<0,05)$ que aquelas que dispunham de coleta regular ou irregular de resíduos sólidos (Tabela 6).

\section{Conclusão}

Os resultados do estudo mostram com alguma evidência uma associação estatisticamente significante entre o tipo de acondicionamento domiciliar dos resíduos sólidos, bem como entre a coleta de resíduos sólidos domiciliares no ambiente de domínio público e a prevalência de $A$. lumbricoides, T. trichiura e ancilostomídeos em crianças entre 5 e 14 anos de idade, mesmo quando outros fatores sócio-econômicos, culturais, demográficos e ambientais são considerados, e a incidência de diarréia e o estado nutricional das crianças menores de cinco anos residentes em assentamentos periurbanos de Salvador.

Desse modo, os resultados sugerem que tanto medidas de domínio doméstico como o tipo de acondicionamento domiciliar, quanto medidas de domínio público como a prestação de serviço de coleta domiciliar regular de resíduos sólidos, contribuíram para controlar a transmissão das
Tabela 5

Coleta de resíduos sólidos domiciliares e incidência de diarréia $(n=1.017)$.

\begin{tabular}{|c|c|c|}
\hline Coleta de resíduos sólidos & $\%$ & $\begin{array}{c}\text { Episódios por criança/ } \\
\text { ano } *, \star \star\end{array}$ \\
\hline Regular & 31,3 & 1,77 \\
\hline Irregular & 30,3 & $2,86 * \star \star * \#$ \\
\hline Não coletado & 38,4 & 5,04 * \\
\hline
\end{tabular}

* $\mathrm{p}<0,001$;

** Análise de variância unidirecional usada para comparação de incidências médias: valor de p apresentado abaixo;

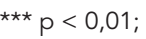

\# Significância da diferença de incidência em relação ao nível menos exposto.

doenças estudadas deixando, porém, um residual que depende de outros fatores de risco.

A universalização do serviço de coleta regular de resíduos sólidos domiciliares torna-se então necessária, visando a contribuir para a redução do quadro de morbidade das crianças residentes em áreas carentes deste importante serviço de saneamento ambiental. Importância também deve ser dada à educação sanitária e ambiental visando à sua contribuição para o processo de mudança de atitudes e práticas das pessoas quanto ao manejo e acondicionamento domiciliar dos resíduos sólidos.

Tabela 6

Coleta de resíduos sólidos domiciliares e estado nutricional $(n=1.074)$.

\begin{tabular}{lccc}
\hline Coleta de resíduos sólidos & Altura/ldade & $\begin{array}{c}\text { Indicadores antropométricos } \\
\text { Peso/ldade }\end{array}$ & Peso/Altura \\
\hline Regular & $-0,51$ & $-0,33$ & $-0,04$ \\
Irregular & $-0,64$ & $-0,57$ & $-0,20$ \\
Não coletado & $-1,04$ & $-0,65$ & $-0,06$ \\
Valor de p* & $<0,001$ & $<0,05$ & $\star \star$ \\
\hline
\end{tabular}

* Análise de variância unidirecional usada para comparação de médias;

** Não significante $(p>0,05)$. 


\section{Resumo}

O artigo apresenta estudo do acondicionamento e coleta de resíduos sólidos domiciliares e o impacto na saúde de crianças, por meio de pesquisa realizada em nove assentamentos humanos localizados em área periurbana da cidade de Salvador, Bahia, Brasil. Como indicadores epidemiológicos foram utilizados, em 1.893 crianças entre 5 e 14 anos, a infecção por nematóides intestinais, expressa pela prevalência de Ascaris lumbricoides, Trichuris trichiura e ancilostomídeos, $e$ em 1.204 crianças menores de cinco anos, a incidência de diarréia e o estado nutricional, este expresso por indicadores antropométricos. Os resultados apresentam a maior prevalência dos três nematóides nas crianças dos domicílios que não dispõem de acondicionamento adequado e de coleta de resíduos sólidos que naquelas de domicílios com acondicionamento adequado e coleta regular, sendo a diferença encontrada estatisticamente significante, mesmo quando outros fatores de risco sócio-econômicos, culturais, demográficos e ambientais são considerados. Resultado semelhante é também observado com relação aos indicadores epidemiológicos, incidência de diarréia e estado nutricional.

Diarréia; Infecções por Nematóides; Acondicionamento de Resíduos Sólidos; Coleta de Resíduos Sólidos

\section{Referências}

1. Tchobanoglous G, Theisen H, Vigil SA. Integrated solid wastes: engineering principles and management issues. New York: McGraw-Hill; 1993.

2. Schmid AW. Aspectos epidemiológicos ligados ao lixo. In: Anais do Seminário sobre o Problema do Lixo no Meio Urbano. São Paulo: Faculdade de Saúde Pública, Universidade de São Paulo/Organização Pan-Americana da Saúde; 1965. p. 9-20.

3. Forattini OP. Aspectos epidemiológicos ligados ao lixo. In: Faculdade de Saúde Pública, Universidade de São Paulo/Organização Pan-Americana da Saúde, organizadores. Lixo e limpeza pública. São Paulo: Universidade de São Paulo; 1969. p. 3.13.19 .

4. Oliveira WE. Resíduos sólidos e poluição ambiental. Rev DAE 1975; 75:46-56.

5. Rocha AA, Lindenberg RC. Impacto dos resíduos sólidos urbanos. In: Companhia de Tecnologia de Saneamento Ambiental, organizador. Resíduos sólidos e limpeza pública. São Paulo: Companhia de Tecnologia de Saneamento Ambiental; 1990. p. 1-13.
6. Bertussi Filho LA. Curso de resíduos sólidos de serviços de saúde: gerenciamento, tratamento e destinação final. Curitiba: Associação Brasileira de Engenharia Sanitária e Ambiental; 1994.

7. Fundação Nacional de Saúde. Manual de saneamento. 3ạ Ed. Brasília: Fundação Nacional de Saúde; 2004.

8. Organización Panamericana de la Salud. La eliminación de basuras y el control de insectos y roedores. Washington DC: Organización Panamericana de la Salud; 1962. (Publicación Científica, 75).

9. Zanon U. Riscos infecciosos imputados ao lixo hospitalar. Realidade epidemiológica ou ficção sanitária? Rev Soc Bras Med Trop 1990; 23:163-70.

10. Hanks TG. Solid waste/disease relationships. Cincinnati: U.S. Department of Health, Education and Welfare/Public Health Services; 1967.

11. Catapreta CAA. Associação entre coleta de resíduos sólidos domiciliares e indicadores de saúde em vilas e favelas de Belo Horizonte [Dissertação de Mestrado]. Belo Horizonte: Escola de Engenharia, Universidade Federal de Minas Gerais; 1997. 
12. Moraes LRS. Impacto na saúde do acondicionamento e coleta dos resíduos sólidos domiciliares. In: Anais do XXVI Congresso Interamericano de Ingeniería Sanitaria y Ambiental [CD-ROM]. Lima: Asociación Interamericana de Ingenería Sanitaria y Ambiental; 1998.

13. Rêgo RCF. Resíduos sólidos domiciliares urbanos e diarréia infantil em Salvador [Tese de Doutorado]. Salvador: Instituto de Saúde Coletiva, Universidade Federal da Bahia; 2002.

14. Moraes LRS. Health impact of drainage and sewerage in poor urban areas in Salvador, Brazil [Tese de Doutorado]. London: London School of Hygiene and Tropical Medicine, University of London; 1996.

15. Katz N, Chaves A, Pellegrino J. A simple device for quantitative stool thick-smear technique in Schistosomiasis mansoni. Rev Inst Med Trop São Paulo 1972; 14:397-400.
16. Heller L. Associação entre cenários de saneamento e diarréia em Betim-MG: o emprego do delineamento epidemiológico caso-controle na definição de prioridades de intervenção [Tese de Doutorado]. Belo Horizonte: Escola de Veterinária, Universidade Federal de Minas Gerais; 1995.

17. Catapreta CAA, Heller L. Associação entre coleta de resíduos sólidos domiciliares e saúde, em Belo Horizonte (MG), Brasil. Rev Panam Salud Pública 1999; 5:88-96.

18. Rego RF, Moraes LRS, Dourado I. Diarrhoea and garbage disposal in Salvador, Brazil. Trans R Soc Trop Med Hyg 2005; 99:48-54.

Recebido em 02/Mai/2006

Versão final reapresentada em 17/Ago/2006

Aprovado em 06/Nov/2006 